\title{
OPORTUNIDAD PARA APROVECHAMIENTO DE PLANTACIONES FORESTALES EN LA SIERRA DEL PERÚ
}

\author{
OPPORTUNITY TO DEVELOP A COMMUNITY FOREST INDUSTRY FROM PLANTATIONS AT THE ANDES ON \\ PERU
}

José Portocarrero G. ${ }^{1}$, Héctor Cahuas M. ${ }^{2}$

https://doi.org/10.52109/cyp2022327

${ }^{1}$ Magister en Conservación de Recursos Forestales. Profesor visitante de la Facultad de Ciencias Forestales de la Universidad Nacional Agraria La Molina.

2 Ingeniero Forestal, Universidad Nacional Agraria La Molina

\section{REGISTROS}

Recibido el 16/10/2021

Aceptado el 27/01/2022

Publicado el 31/01/2022

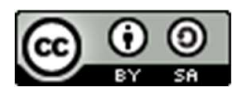

PALABRAS CLAVE

Pinus radiata, plantaciones, Andes

KEYWORDS

Pinus radiata, plantations, Andean

\section{RESUMEN}

El presente artículo muestra la búsqueda bibliográfica relacionada a la competitividad de las importaciones de pino chileno (Pinus radiata) y su incidencia en el mercado de la fabricación de productos de madera en el mercado peruano. Asimismo, presenta la oportunidad de implementar de pequeñas industrias forestales conformadas a partir del aprovechamiento de plantaciones forestales, en especial de pino, llevadas a cabo en las comunidades de la sierra del departamento del Cuzco. El estudio se ha realizado con información secundaria y primaria

\begin{abstract}
This article shows the literature search related to the competitiveness of imports worldwide of pine (Pinus radiata), specially from Chile, and its impact on the market for the manufacture of wood products in the Peruvian market. Likewise, it presents the opportunity to implement updated forestry inventories for implementation of small forest industries formed from the use of forest plantations, especially pine, carried out in the communities of the mountains of the department of Cuzco. This review has been carried out with secondary and primary information.
\end{abstract}

\section{INTRODUCCIÓN}

\section{Plantaciones forestales en la sierra del Perú}

De acuerdo con Guariguata, Arce (2016), en los últimos años ha emergido la voluntad política del Gobierno peruano de apostar al sector forestal como uno de los nuevos motores de la economía en la lucha contra la pobreza, la deforestación del bosque natural y el cambio climático global. En este sentido, se reconoce que las plantaciones forestales son una gran oportunidad de negocio, generación de empleo y recuperación de áreas degradadas. 
En efecto, según fuentes gubernamentales citadas por la Agencia Andina (2021) se ha aprobado para el sector forestal un beneficio tributario que implica una rebaja de $15 \%$ del Impuesto a la Renta hasta el 2030, para todas las empresas con ingresos netos menores a 1,700 UIT, cerca de 7.5 millones de soles". Asimismo, señala dicha fuente que "Por diversas razones este sector estaba pagando 30\% de Impuesto a la Renta de sus ventas totales, una cantidad tan alta que prácticamente lo hacía no competitivo, o lo empujaba a la informalidad".

EI SERFOR (Servicio Nacional Forestal y de Fauna Silvestre [SERFOR], 2018) indica que para 2018 en un inventario realizado para la zona del Cusco, en los distritos de Ancahuasi, Anta, Cachimayo, Chinchaypujio, Chinchero, Huarocondo, Huayllabamba, Paucartambo, Pucyura, Urcos, Urubamba y Zurite, donde se registraron las especies de Eucalyptus globulus, Escallonia resinosa, Pinus radiata y Alnus acuminata con un volumen total para el caso del Eucalyptus globulus de $452.345 \mathrm{~m} 3 / \mathrm{ha}$ la especie con mayor demanda en las comunidades campesinas.

Las 258 unidades muestrales del inventario de plantaciones forestales del departamento de Cusco se encontraban distribuidas en 21 comunidades campesinas y en 70 plantaciones forestales.

El Servicio Nacional Forestal y de Fauna Silvestre (SERFOR, 2021), indica que para el aprovechamiento de las plantaciones forestales es un requisito el registro de dicha plantación ante la autoridad de flora y fauna silvestre correspondiente. Asimismo, las actividades de aprovechamiento son: planificación, corta, trozado y apilado, arrastre, carga y descarga, transporte, transformación y comercialización.

Agrorural (2018) en un estudio realizado para la cadena de valor de productos forestales en la sierra de la Libertad, indica que se deben revisar modelos exitosos de sectores forestales en otros países y adquirir aspectos y programas aplicables a la región. Asimismo, realizan un análisis completo desde la plantación, manejo, aprovechamiento y comercialización de productos forestales provenientes de las plantaciones realizadas bajo el marco del proyecto PP0130. Como dato importante indican que el crecimiento anual de una plantación de pino radiata es alrededor de $12 \mathrm{~m} 3 / \mathrm{Ha}$, y al final del turno de rotación de 22 años, se cosechan alrededor de 288 $\mathrm{m} 3 / \mathrm{Ha}$. Cabe destacar que este rendimiento es con bajo o nulo manejo de la plantación. Por la venta de esa madera obtienen s/80,000, es decir de madera en rollos que es enviada y comercializada en Trujillo, esto constituye que el M3 de madera rolliza puesta en Trujillo sea de S/278 aproximadamente.

Fisher (2018) indica como uno de los requerimientos el modelo de asociatividad con CCNN o comunidades campesinas. Asimismo, se estima que de las 500,000 has existentes en el Perú tan solo 50,000 has son para uso industrial (aserrío, producción puertas, etc.) y el resto para uso local (pobladores). Asimismo, indica que el $90 \%$ de importaciones de madera son productos con valor agregado.

Bermúdez y Dobbertin (2018), en un estudio de costos para 2 bloques de plantación en Porcón, determinaron que el rendimiento por hectárea luego de 22 años y sin 
ningún tipo de manejo intermedio, fluctuaba entre $339 \mathrm{m3} / \mathrm{ha}$ (bloque Lomo de pescado de 47 has) y 599 m3/ha (bloque Cushuro de 22 has). Los productos que se obtendrían en cada bloque son: Tableros de partículas (79.6\% en lomo de pescado y $68.6 \%$ en Cushuro), parihuelas (17.2\% en Lomo de Pescado y $17.5 \%$ en Cushuro), aserrío (3.1\% en Lomo de pescado y $13.9 \%$ en Cushuro). Como se puede apreciar, el bloque Cushuro a pesar de tener una menor superficie tiene un mayor rendimiento que el bloque Lomo de Pescado.

En la tabla $\mathrm{N}^{\circ} 1$ se muestran los costos de aprovechamiento por cada bloque. Cabe destacar que mientras el área a aprovechar sea menor los costos se incrementan.

Tabla 1

Costos de aprovechamiento por bloque

\begin{tabular}{cccc}
\hline Bloque & Área & Costo/Ha (S/) & Costo Total (S/) \\
\hline Lomo de pescado & 47 & 12570.35 & 590806.45 \\
Cushuro & 29 & 18773.08 & 544419.32 \\
\hline
\end{tabular}

El Servicio Nacional Forestal y de Fauna Silvestre (SERFOR, 2019) indica que el mercado actual se está nutriendo de plantaciones ya existentes con poco manejo y baja calidad, es necesario mejorar las condiciones de transformación primaria para brindar mayores oportunidades para la madera del productor. Actualmente los aserraderos existentes están destinados a cortes y trozados gruesos, no hay tecnología para obtener productos específicos de nichos de mercado específicos. La cadena productiva forestal se ve limitada en cuanto al acceso y conocimiento de tecnología e investigación referida no sólo a las especies y productos que el mercado demanda, sino a los tiempos en los que esta tecnología debiera darse en el sector. Asimismo, para invertir en el sector forestal es el poco acceso a mecanismos de financiamiento que permitan aminorar los costos iniciales de la inversión y sostener la misma durante el tiempo que demora la cadena (6-25 años).

\section{Mercado de madera-transformación}

Bardales y García (2018), en un estudio realizado para utilizar residuos de la producción de la línea de aserrío en la empresa DERIMA S.R.L. en Cajamarca usando mecanismos de producción limpia. Los principales productos de esta empresa son madera aserrada para parihuelas, muebles, puertas, mangos para herramientas entre otros. De este estudio se puede concluir que si es posible utilizar los residuos del aserrío debido al volumen generado que corresponde a un 30\% de cortezas y aserrín. El ingreso marginal por la venta de residuos, en especial aserrín es muy bajo (S/ 0.1 por kg, produce $3500 \mathrm{~kg}$ mensual).

Los principales competidores de venta de tableros aglomerados son empresas como Sodimac, Promart y Maestro. Después de haber realizado el desarrollo de la metodología se obtuvo que la empresa incrementa en un 12\% los ingresos, un 
margen de contribución del $27.7 \%$ y un $20 \%$ en cuanto a la satisfacción del cliente. Adicionalmente se evidenció que un $25 \%$ de residuos generados por la actividad serán utilizados en su totalidad para la línea de producción de tableros aglomerados.

Vidal (2021) indica una serie de factores que no permiten obtener el abastecimiento y calidad suficientes para que el Pino que pueda obtenerse de plantaciones forestales en el Perú, sea atractivo para el mercado nacional, estos factores son:

- Volúmenes insuficientes para abastecer la demanda del mercado, sobre todo de madera aserrada.

- Informalidad en los compromisos de abastecimiento de determinados volúmenes de madera aserrada. Esto involucra que muchos productores de madera no estén legalmente constituidos.

- Falta de instalación de plantas de transformación apropiadas que cuenten con hornos de secado para que el producto sea atractivo a posibles clientes y pueda competir con pino proveniente de Chile.

- Por otro lado, la ventaja del Pino importado de Chile ofrece volúmenes suficientes de madera seca al horno y en medidas comerciales (3X4, 4X4), el costo del precio de este pino a raíz de la crisis sanitaria el último año ha subido de USD 0.7-pt a 1-1.2 USD/pt es decir se ha incrementado entre $0.3 \mathrm{y}$ 0.5 USD por pt, por consiguiente suben los costos y precio de venta de los productos que usan este insumo, especialmente las dedicadas a la fabricación de Pallets para la agroexportación. Asimismo, hay competencia de madera de plantaciones de maderas tropicales provenientes de la selva, con especies como bolaina, capinurí y catahua, las cuales en algunos casos mezclan con el pino para la fabricación de pallets.

El diseño de la estrategia de comercialización como prospección consistiría en venta de madera predimensionada para la fabricación de parihuelas y madera aserrada para carpintería y construcción.

\section{Alcance del estudio}

El alcance del estudio ha sido consignado a todas las importaciones de pino radiata provenientes de Chile y otros países entre 2016 y 2020, así como a su importancia en el balance comercial y la oportunidad de competencia que pueda generarse con plantaciones de Pino establecidas en el Cusco desde 2009 en adelante.

\section{MATERIALES Y MÉTODOS}

En el presente trabajo se ha utilizado Información sobre importaciones y exportaciones de madera aserrada de pino. Se seleccionó información obtenida de la web con relación a la importación que realiza el Perú de madera aserrada de Pino radiata (Pinus radiata), en primer lugar, a nivel general y luego específicamente de la proveniente de Chile, teniendo en cuenta las características de esta. La información corresponde al período comprendido entre los años 2016 al 2020. Asimismo, se hizo un breve análisis del potencial que podrían generar las 
plantaciones provenientes de 4 comunidades del Cusco que cuentan con edades mayores a 08 años y podrían ser raleadas.

\section{RESULTADOS}

En la tabla 2, se puede observar el comportamiento de las exportaciones de madera aserrada y los principales destinos de esta. Por otro lado, en la tabla 3, se observa que las importaciones de madera aserrada en su mayoría provienen de Chile.

Tabla 2

Principales destinos de exportación de madera aserrada

\begin{tabular}{|c|c|c|c|c|c|}
\hline Importadores & $\begin{array}{c}\text { Valor } \\
\text { exportado } \\
\text { en } 2016\end{array}$ & $\begin{array}{c}\text { Valor } \\
\text { exportado } \\
\text { en } 2017\end{array}$ & $\begin{array}{c}\text { Valor } \\
\text { exportado } \\
\text { en } 2018\end{array}$ & $\begin{array}{c}\text { Valor } \\
\text { exportado } \\
\text { en } 2019\end{array}$ & $\begin{array}{c}\text { Valor } \\
\text { exportado } \\
\text { en } 2020\end{array}$ \\
\hline Mundo & 27859 & 24441 & 22539 & 26554 & 23978 \\
\hline China & 9921 & 5336 & 4884 & 6579 & 9408 \\
\hline $\begin{array}{l}\text { República } \\
\text { Dominicana }\end{array}$ & 6053 & 7725 & 6816 & 8679 & 4862 \\
\hline México & 4921 & 6848 & 5270 & 6664 & 3700 \\
\hline Viet Nam & 0 & 79 & 1278 & 1015 & 2839 \\
\hline Ecuador & 129 & 19 & 0 & 0 & 1288 \\
\hline Estados & & & & & \\
\hline $\begin{array}{l}\text { Unidos de } \\
\text { América }\end{array}$ & 2634 & 1659 & 1350 & 1019 & 494 \\
\hline $\begin{array}{l}\text { Corea, } \\
\text { República de }\end{array}$ & 462 & 354 & 466 & 539 & 440 \\
\hline Francia & 92 & 23 & 70 & 38 & 237 \\
\hline Dinamarca & 41 & 84 & 28 & 0 & 128 \\
\hline Países Bajos & 779 & 442 & 164 & 0 & 90 \\
\hline $\begin{array}{l}\text { Nueva } \\
\text { Zelandia }\end{array}$ & 57 & 176 & 518 & 256 & 76 \\
\hline Chile & 183 & 227 & 298 & 192 & 60 \\
\hline Australia & 361 & 179 & 149 & 212 & 57 \\
\hline Argentina & 33 & 0 & 0 & 0 & 51 \\
\hline Cuba & 106 & 168 & 499 & 1151 & 47 \\
\hline
\end{tabular}




\begin{tabular}{|c|c|c|c|c|c|}
\hline Importadores & $\begin{array}{c}\text { Valor } \\
\text { exportado } \\
\text { en } 2016\end{array}$ & $\begin{array}{c}\text { Valor } \\
\text { exportado } \\
\text { en } 2017\end{array}$ & $\begin{array}{c}\text { Valor } \\
\text { exportado } \\
\text { en } 2018\end{array}$ & $\begin{array}{c}\text { Valor } \\
\text { exportado } \\
\text { en } 2019\end{array}$ & $\begin{array}{c}\text { Valor } \\
\text { exportado } \\
\text { en } 2020\end{array}$ \\
\hline España & 141 & 394 & 365 & 22 & 39 \\
\hline Jamaica & 29 & 0 & 0 & 58 & 30 \\
\hline Filipinas & 75 & 73 & 75 & 0 & 30 \\
\hline Reino Unido & 382 & 312 & 79 & 0 & 23 \\
\hline Canadá & 36 & 51 & 0 & 4 & 21 \\
\hline Barbados & 0 & 0 & 0 & 0 & 18 \\
\hline Uruguay & 66 & 0 & 38 & 0 & 13 \\
\hline Alemania & 104 & 47 & 2 & 0 & 12 \\
\hline $\begin{array}{l}\text { Rusia, } \\
\text { Federación de }\end{array}$ & 0 & 0 & 0 & 0 & 11 \\
\hline Guatemala & 26 & 147 & 6 & 9 & 4 \\
\hline Bahamas & 15 & 0 & 0 & 0 & 0 \\
\hline Bélgica & 553 & 0 & 29 & 22 & 0 \\
\hline Costa Rica & 59 & 0 & 0 & 4 & 0 \\
\hline Croacia & 0 & 14 & 0 & 0 & 0 \\
\hline Chipre & 0 & 0 & 11 & 0 & 0 \\
\hline Haití & 0 & 0 & 0 & 59 & 0 \\
\hline $\begin{array}{l}\text { Hong Kong, } \\
\text { China }\end{array}$ & 34 & 0 & 0 & 0 & 0 \\
\hline Indonesia & 20 & 27 & 28 & 31 & 0 \\
\hline Japón & 175 & 31 & 0 & 0 & 0 \\
\hline Luxemburgo & 58 & 0 & 0 & 0 & 0 \\
\hline Mauricio & 0 & 0 & 31 & 0 & 0 \\
\hline Taipei Chino & 278 & 0 & 86 & 0 & 0 \\
\hline Marruecos & 38 & 0 & 0 & 0 & 0 \\
\hline Polonia & 0 & 25 & 0 & 0 & 0 \\
\hline
\end{tabular}


Tabla 3

Importaciones de madera aserrada entre 2016 y 2020

\begin{tabular}{|c|c|c|c|c|c|}
\hline Exportadores & $\begin{array}{c}\text { Valor } \\
\text { importado en } \\
2016\end{array}$ & $\begin{array}{c}\text { Valor } \\
\text { importado en } \\
2017\end{array}$ & $\begin{array}{c}\text { Valor } \\
\text { importado en } \\
2018\end{array}$ & $\begin{array}{c}\text { Valor } \\
\text { importado en } \\
2019\end{array}$ & $\begin{array}{c}\text { Valor } \\
\text { importado en } \\
2020\end{array}$ \\
\hline Mundo & 28.085 & 30.508 & 37.768 & 36.571 & 36.010 \\
\hline Chile & 22.999 & 24.929 & 29.874 & 28.434 & 29.006 \\
\hline $\begin{array}{l}\text { Estados Unidos } \\
\text { de América }\end{array}$ & 1.763 & 2.002 & 2.507 & 3.094 & 1.967 \\
\hline Brasil & 632 & 1.075 & 2.513 & 1.481 & 1.741 \\
\hline $\begin{array}{l}\text { Venezuela, } \\
\text { República } \\
\text { Bolivariana de }\end{array}$ & 391 & 776 & 950 & 879 & 1.143 \\
\hline Argentina & 0 & 0 & 0 & 1.234 & 860 \\
\hline Uruguay & 1.720 & 1.156 & 543 & 711 & 672 \\
\hline Canadá & 168 & 317 & 810 & 376 & 447 \\
\hline España & 349 & 174 & 312 & 95 & 84 \\
\hline Ecuador & 0 & 47 & 112 & 87 & 50 \\
\hline Portugal & 0 & 0 & 27 & 80 & 25 \\
\hline Italia & 0 & 0 & 0 & 28 & 14 \\
\hline Austria & 3 & 0 & 0 & 0 & 0 \\
\hline Myanmar & 29 & 23 & 0 & 5 & 0 \\
\hline China & 1 & 0 & 10 & 0 & 0 \\
\hline Dinamarca & 0 & 0 & 0 & 0 & 0 \\
\hline Francia & 0 & 0 & 0 & 47 & 0 \\
\hline Honduras & 30 & 0 & 30 & 0 & 0 \\
\hline
\end{tabular}




\begin{tabular}{|c|c|c|c|c|c|}
\hline Exportadores & $\begin{array}{c}\text { Valor } \\
\text { importado en } \\
2016\end{array}$ & $\begin{array}{c}\text { Valor } \\
\text { importado en } \\
2017\end{array}$ & $\begin{array}{c}\text { Valor } \\
\text { importado en } \\
2018\end{array}$ & $\begin{array}{c}\text { Valor } \\
\text { importado en } \\
2019\end{array}$ & $\begin{array}{c}\text { Valor } \\
\text { importado en } \\
2020\end{array}$ \\
\hline Côte d'Ivoire & 0 & 0 & 42 & 0 & 0 \\
\hline México & 0 & 9 & 0 & 0 & 0 \\
\hline Paraguay & 0 & 0 & 30 & 0 & 0 \\
\hline Polonia & 0 & 0 & 0 & 20 & 0 \\
\hline India & 0 & 0 & 9 & 0 & 0 \\
\hline
\end{tabular}

Nota: Fuente, International Trade Centre - [ITC]. (2021a). Trade statistics for international business development. List of supplying markets for the product exported by Peru in 2020 Metadata. Product: 4407

Figura 1

Exportaciones e importaciones de madera aserrada entre los años 2016 y 2020

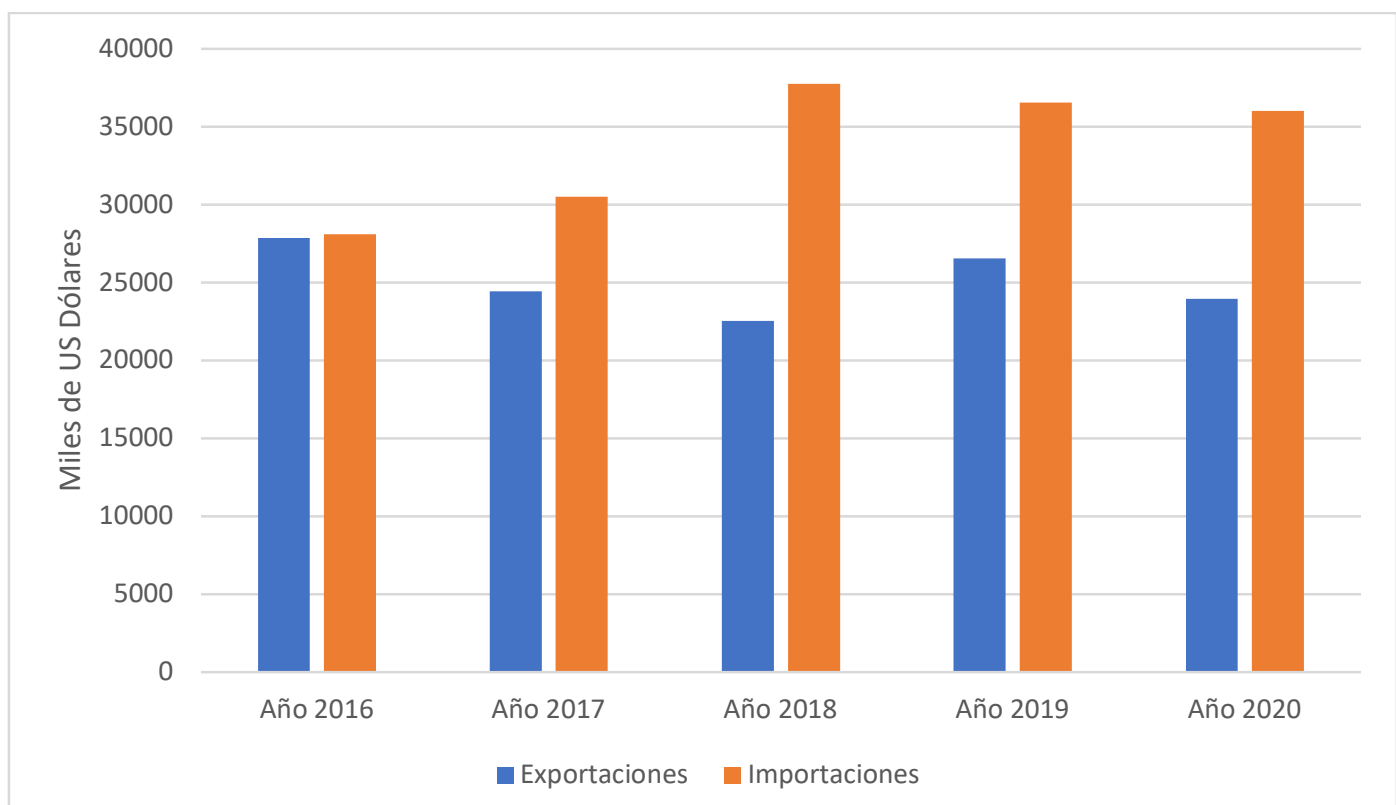

Nota: Fuente; International Trade Centre - [ITC]. (2021). Trade statistics for international business development.

\section{DISCUSIÓN}

La presente evaluación de comercialización se fundamenta en la información disponible respecto al manejo y aprovechamiento de plantaciones forestales en diversas zonas de la sierra del Perú y en el mercado nacional dirigido al consumo interno de madera de pino radiata especialmente. 
La cadena productiva del pino radiata de origen nacional se basa en la venta de trozas a aserraderos o pequeños productores y la venta de madera aserrada producida por algunas comunidades como es el caso de Porcón en el departamento de Cajamarca. Asimismo, se tiene como principal producto de comercialización la madera en trozas dirigida para el mercado de parihuelas (pallets), madera aserrada y otros productos como postes, son muy pocos los datos de comunidades que den valor agregado a sus plantaciones. Cabe destacar que la mayoría utiliza tecnología básica para el aprovechamiento de la plantación, así como no dan el debido manejo técnico de la plantación para que los niveles de producción sean atractivos para inversionistas. Por ejemplo, el uso de tecnología intermedia, un correcto diseño de productos, marca con denominación de origen y socios estratégico-comerciales garantizarían una alta probabilidad de sostenibilidad y rentabilidad de las plantaciones.

Es necesario evaluar y analizar la dispersión y fragmentación de las plantaciones existentes y el efecto en el aprovechamiento de las mismas. Asimismo, es necesario identificar y cuantificar como afecta el escaso manejo de las plantaciones a su sostenibilidad y competitividad.

Muchas plantaciones forestales han sido instaladas en tierras de protección empleando especies que no necesariamente reúnen las bondades para dicho objetivo ecosistémico, ocasionando serias deficiencias en la protección, así como en las técnicas de aprovechamiento racional y manejo sostenible, bajos ingresos económicos, entre otros factores que desalientan la actividad.

Es necesario realizar un ordenamiento de las futuras y actuales plantaciones, así como la identificación de mercados y productos (y sus respectivos paquetes tecnológicos) que permitan garantizar la sostenibilidad y competitividad de dichas plantaciones bajo un modelo específico de producción (asociativo, comunal, etc). Asimismo, de acuerdo con la información proporcionada por Pachamama Raymi, la realización de un inventario actualizado en las comunidades con mayor cantidad de árboles plantado brindaría información necesaria para implementar un plan piloto de aprovechamiento de dichas plantaciones.

\section{CONCLUSIONES}

El potencial de aprovechamiento de plantaciones forestales de pino en los andes, carece de estudios suficientes para definir una oferta comercial realista que permita competir con la madera aserrada importada proveniente de Chile. Una oportunidad para iniciar estos estudios es en las plantaciones del Cusco que realizó Pachamamaraymi.

La balanza comercial respecto a madera aserrada es negativa. Chile es el principal abastecedor de madera aserrada de pino, seca y predimensionada en el Perú. 
Un factor que afecta al mercado de la madera es la informalidad, pues se utiliza madera aserrada que no cuenta con el proceso legal para la fabricación de productos maderables, los cuales son vendidos en mercados informales a menor precio que los provenientes de concesiones o plantaciones legalmente establecidas.

\section{REFERENCIAS}

Agencia andina. (29 de diciembre de 2021) Gobierno aprueba beneficios tributarios para sectores acuícola, forestal y de atún. https://andina.pe/agencia/noticiagobierno-aprueba-beneficios-tributarios-para-sectores-acuicola-forestal-yatun-875131.aspx

Agrorural (2018). “Línea de Base y el Análisis de los Procesos productivos y Cadena de Valor de las Plantaciones Forestales en la Región La Libertad". 158pag.

Bardales, M., García M., (2018) Diseño de una línea de producción de tableros aglomerados aplicando la estrategia de producción más limpia y su relación con el nivel de competitividad en la empresa DERIMA s.r.l. Tesis para optar el título de ingeniero Industrial. Universidad Privada del Norte-Facultad de Ingeniería-Carrera de Ingeniería Industrial. 69 p.

Bermúdez, S. (2018) Análisis de costos de aprovechamiento en primer raleo de una plantación de pinos en la Granja Porcón, Cajamarca-Perú. Tesis para optar el título de Ingeniero Forestal.Universidad nacional Agraria La Molina-facultad de Ciencias Forestales. Lima. 67p.

FAO, ITP, CITE Madera. (2018) La industria de la Madera en el Perú-Identificación de las barreras y oportunidades para el comercio interno de productos responsables de madera, provenientes de fuentes sostenibles y legales, en las MIPYMEs del Perú.178 p.

Fisher (2019). Agenda pendiente del sector forestal- Asociación de Exportadores ADEX. Presentación. https://www.bcrp.gob.pe/docs/Proyeccion-Institucional/EncuentrosRegionales/2019/cajamarca/eer-cajamarca-2019-fischer.pdf . 26 p.

Guariguata. M.R, Arce,J., Ammour T., Capella, J.L (2017). Las plantaciones forestales en Perú Reflexiones, estatus actual y perspectivas a futuro. Documento ocasional 169.CIFOR. 40p.

International Trade Centre - [ITC]. (2021a). Trade statistics for international business development. List of supplying markets for the product imported by Peru in 2020 Metadata. Product: 4415 Packing cases, boxes, crates, drums and similar packings, of wood; cable-drums of wood; pallets, box pallets and other load boards, of wood; pallet collars of wood (excluding containers specially designed and equipped for one or more modes of transport). https://www.trademap.org/Country_SelProductCountry_TS.aspx?nvpm=1\%7c 604\%7c\%7c\%7c\%7c441520\%7c\%7c\%7c6\%7c1\%7c1\%7c1\%7c2\%7c1\%7c2\%7c1 $\% 7 c 1 \% 7 c 1$

Pachamama Raymi (2021). Información de plantaciones de pino sembradas en el departamento del Cusco desde 2010. 
Servicio Forestal y de Fauna Silvestre-SERFOR (2019) Estudio de pre-inversión a nivel de perfil de "Fomento y Gestión Sostenible de la Producción Forestal en el Perú" Código único 2332872. 458p.

Servicio Forestal y de Fauna Silvestre-SERFOR-Proyecto de inversión "Alta Productividad del Sector Forestal" (2021) Manual de Manejo Forestal Comunitario en plantaciones forestales. 35p.

Vidal, G. (2021) Entrevista telefónica a Gonzalo Vidal Paulinich, realizada en agosto de 2021. 